\title{
Gigantic Peroxidase Granules in the Leukocytes of the Giant Salamander, Megalobatrachus Japonicus ${ }^{* * * *}$
}

\author{
The 29th Report of Histochemical Study of Peroxidase
}

By

\author{
Tadao Mitsui and Akira Fukui \\ Department of Anatomy, School of Medicine, \\ Keio University, Shinjuku, Tokyo
}

It is of special interest that peculiar and significant findings in blood cells may frequently be obtained with the aid of cytochemical reactions. With the light microscope several studies have been made on cytochemical reactions of blood cells in urodele amphibians (N a k a m u.ra, 1955 (12), S ug i y m a, 1955 (14), Mitsui and

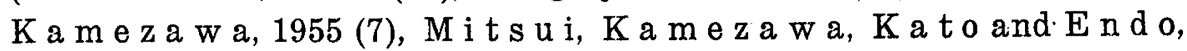
1956 (8), Kat o, 1957 (6), End o, 1958 (2), Mits u i, 1958 (9)). In 1960 , Mitsui (10) already published a paper on cytochemical peroxidase reaction in salamander leukocytes at the level of the electron microscope. A review of the literatures indicates that the neutrophil leukocytes in salamander show peroxidase activity without exceptions, and that the eosinophil leukocytes in salamander do not show peroxidase activity with minor exceptions. These data are always definite irrespective of sort of the substrate used for the peroxidase reaction.

It is a general consequence of the peroxidase reaction that, the neutrophil leukocyte, when observed with the light microscope, displays the reaction predominantly in specific granules, which are diffusely distributed in the cytoplasm. During the cytochemical studies of blood cells in 70 different animals from mammals through to fishes the authors happened to find peculiar neutrophil leukocytes in the giant salamander, which were characterized by an intracellular, single gigantic granule reactive to the peroxidase reagent.

* To the memory of the late Prof. Tsunetaro Fujita.

** The research reported in this document has been made possible through the support and sponsorship of the U.S. Department of Army through its Far East Research Office. 
This type of leukocyte has never been found in any other animals examined.

In the present paper the authors wish to introduce these peculiar neutrophil leukocytes in the giant salamander and discuss the difference from the gigantic peroxidase granules detected in man and rabbit with the copper-benzidine peroxidase method, which have already been published by $\mathrm{Hig}$ a s i (3) and $\mathrm{S}$ a to (13).

\section{Materials and methods}

Two giant salamanders, Megalobatrachus japonicus were used for materials. One was examined in winter, another was in summer. The blood was carefully taken from the tail and smear preparations were obtained. The blood smears were fixed one minute with ethanol-formol $(9: 1)$ solution prior to staining, washed thoroughly in distilled water, and stained five minutes with either of the following peroxidase reagents freshly prepared each time.

1) Mixture of benzidine $0.3 \mathrm{gr}$, $3 \% \mathrm{H}_{2} \mathrm{O}_{2} 10 \mathrm{drops}$, and distilled water $100 \mathrm{cc}$. This mixture should be filtered because of poor solubility of benzidine in water.

2) Mixture of orthophenylenediamine $0.1 \mathrm{gr} ., 3 \% \quad \mathrm{H}_{2} \mathrm{O}_{2} 10 \mathrm{drops}$, and distilled water $100 \mathrm{cc}$.

The smears were then washed in water, and counterstained with dilute $\mathrm{G}$ ie $\mathrm{ms}$ a stain for ten to fifteen minutes. Finally they were washed in water, and exposed to air till dry.

The alkaline phosphatase technic (modified technic of the leadsulphide method of $\mathrm{Také} u \mathrm{ch}$ i (15)) was also applied for blood staining.

\section{Observations}

The preparation stained with the benzidine peroxidase reagent, that with the orthophenylenediamine reagent, and that with G i e m s a stain, were simultaneously observed, and compared.

In the giant salamander, the neutrophil leukocytes generally showed peroxidase activity, while the eosinophil and basophil leukocytes did not. The reaction was lacking in all cells of the lymphatic series. As is well known, the general feature of the peroxidase reaction in human leukocytes under the light microscope is that the cytoplasm is diffusely, fine granularly stained except eosinophil leukocytes in which the reaction may take a pattern of prominent coarse granules corresponding to the eosinophil granules. However, 
in the giant salamander examined in winter, a little different findings could be obtained. Namely, two types were differentiated in the neutrophil leukocytes: 1) Type I, which was large in size, weakly reactive to the peroxidase reagent, and possessed a slender stab or two to three slender nuclei in general, 2) Type II, which was small in size, intensely reactive to the peroxidase reagent, and possessed a single, indented or round nucleus (Figs. 7,8 ). In the flowing blood, Type I was found much more frequently than Type II.

In the majority of Type $I$, the authors encountered a very curious finding in the cytoplasmic peroxidase reaction. The reaction positive substance did not take a diffuse pattern, but indicated a single gigantic brown granule which was easily found even under the low power magnification (Figs. 1, 2, 3, 4, 5, 6). This peroxidase granule occurred in the cytoplasm both with the benzidine peroxidase reagent and the orthophenylenediamine peroxidase reagent, in other words, this peroxidase granule could be always detected irrespective of the sort of the substrate used.

The gigantic peroxidase granule of the neutrophil leukocyte was generally distinct in contour, and six to ten $\mu$ in diameter. In some cases, it looked like a large conglomeration of peroxidase reacting substance with obscure contour. The localization of this granule was generally in the center of the leukocyte or close to the more central side of the nucleus. However, in some cases, it was localized in the perinuclear zone or close to the exoplasmic region.

It should be noted that the gigantic granule in the neutrophil leukocyte of Type I, could never be identified with G i e m s a stain, and that no implication of this granule was obtained with the alkaline phosphatase reaction, in which case the cytoplasm was intensely and diffusely stained.

Furthermore, it should be emphasized that the giant salamander found in summer did not show the gigantic peroxidase granule with the benzidine peroxidase reagent nor with the orthophenylenediamine reagent. This problem will be discussed later.

\section{Discussion}

In peroxidase stained preparations, the neutrophil leukocytes are commonly found to contain fine, numerous, scattered granules in the cytoplasm. Contrary to this general finding the neutrophil leukocytes of the giant salamander took a peculiar pattern in which a single large peroxidase granule was found in the center of the 
cytoplasm. This feature resembles the gigantism of the peroxidase granule that was recognized in the $\mathrm{C}$ he $\mathrm{d}$ i a $\mathrm{k}$ (1) and $\mathrm{H}$ i g a s h i 's disease (13). This congenital human disease is characterized by albinismus with photophobia, early death of albinic sibs and peculiar pathological blood picture in which the above-mentioned gigantic granules or inclusion bodies are demonstrated in the leukocytes. In this disease the peroxidase granules gather in a few or several spots to form round clumps or globes not only in the neutrophils but also in the monocytes, eosinophils, myelocytes and the myeloblasts, in other words it is an abnormity affecting all the cells of the myelogenous system. According to $\mathrm{Hig}$ a s hi's description (3), when stained with Gie m s stain, in most of the neutrophils of this patient, there are several round aggregates of the fine violet-pink granules, and among these abnormal aggregates in the cytoplasm, almost none of the specific granules are found to be scattered freely, while, when stained with the peroxidase reagent, the intracytoplasmic peroxidase granules aggregate very compactly forming one to seven spherical or oval masses, each of which is one to three $\mu$ in diameter. Further he described that the lymphocyte of this patient contained a large round granule one to three $\mu$ in diameter that is detectable with Giem s a stain and not at all with the peroxidase reagent. In short, abnormity in the blood cells could be revealed in the $\mathrm{Ch}$ é $\mathrm{diak}$ and $\mathrm{Hi}$ g a s h i 's disease both with $\mathrm{G}$ iemsa and peroxidase stains although the detailed morphological changes did not always run parallel in both the stainings.

Again, $\mathrm{H}$ ig a s h i et al. (4) suggested in the test of alkaline phosphatase reactions in leukocytes of the same patient that large cytoplasmic stained granules or/and unstained vacuoles corresponded to the site of the above-mentioned gigantic peroxidase granules, although the blood smears used were unfortunately very old.

As regards the difference between the gigantic peroxidase granules found in this disease and in the giant salamander described above, it can be summarized that, in the latter, the giant granule was never recognized with $\mathrm{G}$ ie $\mathrm{ms}$ a stain nor with the alkaline phosphatase technic and that, in the latter, the granule was always single in contrast with one to seven in number in the former. However, the size, shape, localization within the cytoplasm and the intense reactivity to the peroxidase reagent were quite similar in both the granules.

The gigantic peroxidase granules have been seldom found among 
animal leukocytes. Mits u i (11) recognized a single, abnormally large peroxidase granule in the cytoplasm of the bichromatophil leukocytes of a snake, although the contour of the granule was quite obscure as compared with that in the giant salamander. Moreover, the blood smear used was not fresh enough to keep the enzyme active.

$\mathrm{Higash}$ i et al. (5) detected gigantic peroxidase granules in the pseudoeosinophil leukocytes of the normal rabbit. These granules, however, are generally much smaller in size and more numerous than those found in the giant salamander.

It is worthy of special comment that the gigantic peroxidase granule could not be demonstrated in the giant salamander in summer, namely, this granule might have been a temporary inclusion body which occurs only under a definite condition of the living body. This problem will require further investigation, for the origin of the gigantic peroxidase granule is still poorly understood at present.

The gigantic peroxidase granule in the giant salamander may be derived from a conglomeration of the peroxidase substance within the leukocyte under abnormal or normal metabolism of protein and other substances in this animal, or may be a product caused by phagocytosis of a peroxidase reacting substance. However, if a phagocytosis phenomenon is assumed in the giant salamander, it remains still unanswered why it occurred only in the neutrophil leukocytes and not at all in other leukocytes such as monocytes.

The nature and the function of the gigantic peroxidase granule in the giant salamander leukocytes, may be unknown at present, nevertheless it was clarified by the present study that cytochemical technics for enzyme may provide a better understanding for cell identification, than by solely morphological criteria.

\section{Summary}

A gigantic peroxidase granule was detected in the polymorphonuclear neutrophil leukocytes of the giant salamander, Megalobatrachus japonicus. Among 70 different animals examined, this gigantic peroxidase granule was found only in the giant salamander in apparently healthy condition. This granule was generally localized in the center of the leukocyte, single in number, six to ten $\mu$ in diameter, and could be demonstrated both with the benzidine peroxidase reagent and the orthophenylenediamine peroxidase reagent. This granule, however, could not be demonstrated with Giem sa 
stain. It is of interest that one of pathological characters of human leukocytes was found in an apparently healthy giant salamander although it remains to be determined in what way this gigantic peroxidase granule developed only in the neutrophil leukocytes of this animal.

The authors wish to appreciate from their whole heart that Mr. Hiroshi Sugiura, B.S. of the Aquarium in the Uéno Zoological Garden, Tokyo, and Prof. Ki yoshi $\mathrm{Hama}$ of the Hiroshima University generously provided the giant salamanders.

\section{References}

1. Chédi a k, M. M.: Revue d'hématologie. $7: 362-367,1952$.

2. En d o, K.: Okajimas Fol. anat. jap. 30: 427-442, 1958.

3. Hig a s hi, O.: Tohoku J. Exp. Med. 59 : 315-332, 1954.

4. Higashi, O., Kagay a, H. and $\mathrm{Hayashi,} \mathrm{T.:} \mathrm{Tohoku} \mathrm{J.} \mathrm{Exp.} \mathrm{Med.} 63$ : 133-136, 1956.

5. Hig a s hi, O., Ni it s u, K. and T a k a h a $\mathrm{h} \mathrm{i,} \mathrm{T.:} \mathrm{Acta} \mathrm{Haematologica} \mathrm{Japonica.}$ 25 : 101-102, 1962.

6. K a to, K.: Okajimas Fol. anat. jap. 29 : 387-402, 1957.

7. Mitsui, T. and $\mathrm{K}$ a me $z$ a w a, S.: Okajimas Fol. anat. jap. $27: 335-343,1955$.

8. Mitsui, T., Ka me zawa, S., Kat o, K. and Endo, K.: Okajimas Fol. anat. jap. $28: 45-58,1956$.

9. Mitsui, T.: Anat. Rec. $130: 341-342,1958$ (Abstract).

10. Mitsui, T.: J. Biophysic. and Biochem. Cytol. $7: 251-260,1960$.

11. Mitsu i, T.: Unpublished.

12. N a k a m u r a, M.: Okajimas Fol. anat. jap. $27: 1-21,1955$.

13. Sa to, A.: Tohoku J. Exp. Med. 61: 201-210, 1955.

14. Sug i y a m a, K. : Okajimas Fol. anat. jap. $27: 197-220,1955$.

15. Takéuchi, T., Ma eda, K., O h shima, J., T a noue, S., O no, S. and Y okota, S.: Tokyo Iji Shinshi. $68: 11-14,1951$. 
Plate 


\section{Explanation of figures}

Figs. 1-3. Neutrophil leukocyte of the giant salamander stained with the benzidine peroxidase reagent. Counterstained with Giemsa stain. A gigantic peroxidase granule is indicated by arrow. This granule frequently appears in the center of the leukocyte (see Fig. 2). The diameter of the granule is 6 to $10 \mu$. RBC (Erythrocyte). $\times 300$.

Figs. 4-6. Neutrophil leukocyte of the giant salamander stained with the orthophenylenediamine peroxidase reagent. Counterstained with Giemsa stain. A gigantic peroxidase granule is indicated by arrow. As shown in Fig. 5, the granule is frequently seen in the center of the leukocyte. $\times 300$.

Fig. 7. Benzidine peroxidase reaction of two different neutrophil leukocytes of the giant salamander, counterstained with Giemsa stain. In these leukocytes, no gigantic peroxidase granule can be recognized. P ( $\mathrm{Pelg}$ e $r$ leukocyte with a peculiar nucleus, showing an intense, diffuse peroxidase reaction in the cytoplasm). N (Neutrophil leukocyte in which ordinary nucleus and diffuse, poorly developed end-product of the reaction are demonstrated). RBC (Erythrocyte). $\times 750$.

Fig. 8. Benzidine peroxidase reaction of the $\mathrm{Pelg}$ er leukocyte of the giant salamander, counterstained with Giemsa stain. Fine granules are diffusely found in the cytoplasm. The gigantic peroxidase granule never occurs in this type of neutrophil leukocytes. $\times 1,100$

Fig. 9. Orthophenylenediamine peroxidase reaction of the neutrophil leukocyte of the giant salamander, counterstained with Giemsa stain. A gigantic peroxidase granule is indicated by arrow. $\times 1,150$.

Fig. 10. Benzidine peroxidase reaction of neutrophil leukocyte of the giant salamander, counterstained with Giemsa stain. A gigantic peroxidase granule is indicated by arrow. The granule is frequently found in the center of the leukocyte like this. It should be noted that this granule is essentially the same as that stained with the orthophenylenediamine peroxidase reagent, and that this granule is entirely lacking in some neutrophil leukocytes as shown in Fig. 7. $\times 1,100$.

Fig. 11. Orthophenylenediamine peroxidase reaction of the neutrophil leukocyte of the giant salamander, counterstained with Giemsa stain. A gigantic peroxidase granule with obscure contour is seen in the cytoplasm (arrow). The other part of the cytoplasm appears finely granular, containing a small vacuole (V). RBC (Erythrocyte). $\times 1,150$. 
Plate I

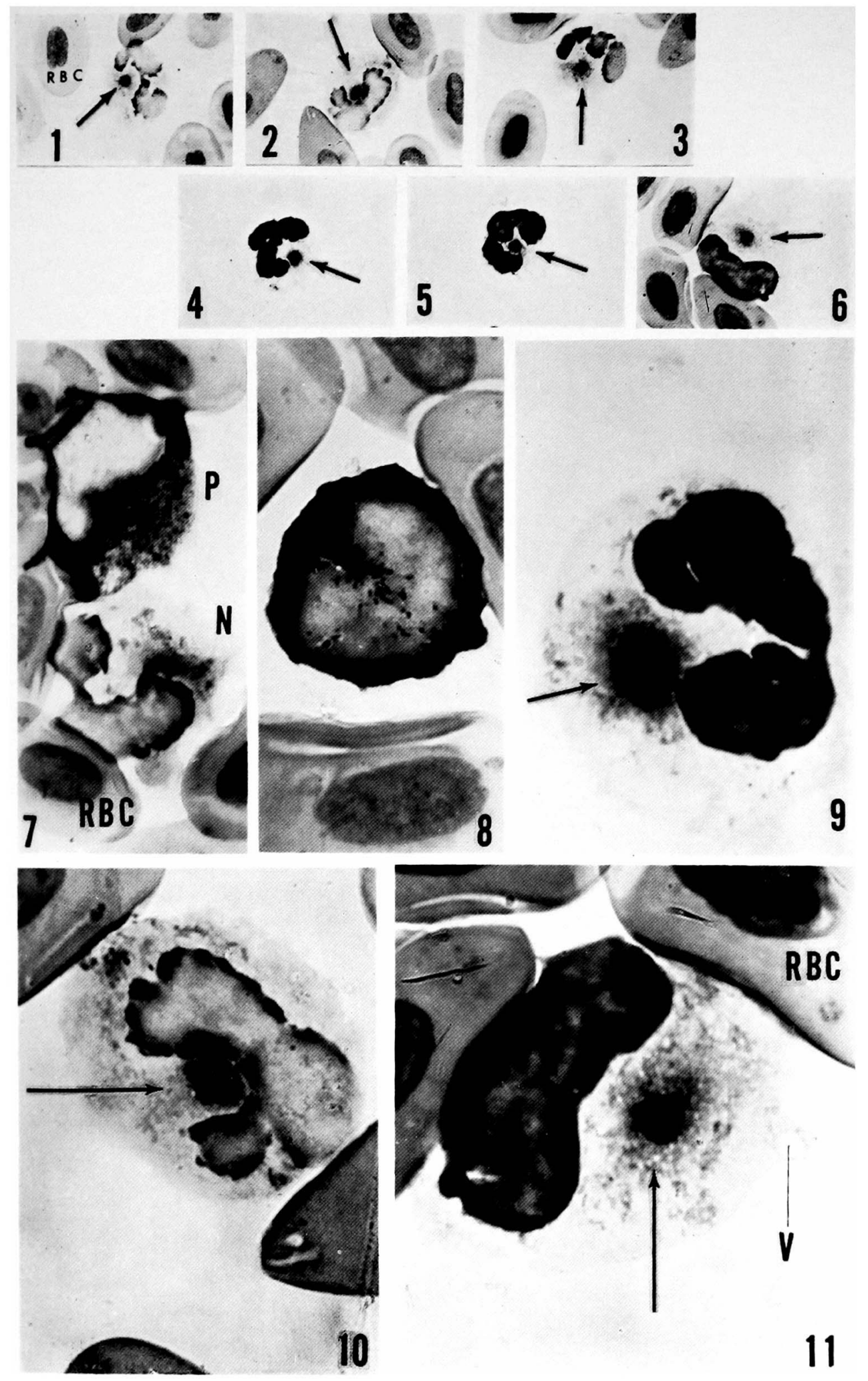

T. Mitsui and A. Fukui 